\title{
ON A CONJECTURE OF POMERANCE
}

\author{
L. HAJDU ${ }^{1}$, N. SARADHA, AND R. TIJDEMAN \\ Dedicated to Professor Schinzel on the occasion of his 75th birthday
}

\begin{abstract}
We say that $k$ is a $P$-integer if the first $\varphi(k)$ primes coprime to $k$ form a reduced residue system modulo $k$. In 1980 Pomerance proved the finiteness of the set of $P$-integers and conjectured that 30 is the largest $P$-integer. We prove the conjecture assuming the Riemann Hypothesis. We further prove that there is no $P$-integer between 30 and $10^{11}$ and above $10^{3500}$.
\end{abstract}

\section{INTRODUCTION}

Let $k>1$ be an integer. We denote Euler's totient function by $\varphi(k)$ and the number of distinct prime divisors of $k$ by $\omega(k)$. We say that $k$ is a $P$-integer if the first $\varphi(k)$ primes coprime to $k$ form a reduced residue system modulo $k$. In 1980, Pomerance [8] proved the finiteness of the set of $P$-integers. The following conjecture was proposed by him in [8].

Conjecture of Pomerance. If $k$ is a $P$-integer, then $k \leq 30$.

This conjecture is still open. Recently, Hajdu and Saradha [3] and Saradha [12] have given simple conditions under which an integer $k$ is not a $P$-integer. By their results, it follows that

- no prime is a $P$-integer except 2 ;

- no square or a cube of a prime is a P-integer except 4;

- no integer $k$ with its least odd prime divisor $>\log k$ is a Pinteger except when $k \in\{2,4,6,12,18,30\}$.

It is easy to check that the only $P$-integers $\leq 30$ are $2,4,6,12,18,30$. It was checked by computation in [3] that if $k$ is another $P$-integer, then $k \geq 5.5 \cdot 10^{5}$. In Theorem 4.1 we improve this bound to $10^{11}$.

2010 Mathematics Subject Classification. Primary 11N13.

Key words and phrases. primes in residue classes, Riemann Hypothesis.

${ }^{1}$ Research supported in part by the OTKA grants K67580 and K75566, and by the TÁMOP 4.2.1./B-09/1/KONV-2010-0007 project. The project is implemented through the New Hungary Development Plan, cofinanced by the European Social Fund and the European Regional Development Fund. 
In this paper, we give a quantitative version of the finiteness result of Pomerance and prove the conjecture of Pomerance under the Riemann Hypothesis. We have

Theorem 1.1. If $k$ is a P-integer, then $k<10^{3500}$.

Theorem 1.2. Suppose the Riemann Hypothesis holds. Then the only P-integers are $2,4,6,12,18,30$.

Pomerance's conjecture is closely related to the classical problem about the least primes in arithmetic progressions. Let $\ell$ be a positive integer with $\operatorname{gcd}(k, \ell)=1$. Denote by $p(k, \ell)$ the least prime $p \equiv$ $\ell(\bmod k)$. Let $P(k)$ be the maximum value of $p(k, \ell)$ for all $\ell$. Linnik [7] has shown that

$$
P(k) \ll k^{L}
$$

for some constant $L$ which is known as Linnik's constant. A huge literature is available on finding good values for $L$ (see $[4,15])$. In the other direction, Prachar [9] and Schinzel [13] have shown that there is an absolute constant $c$ such that for each $\ell$ there are infinitely many $k$ with

$$
p^{\prime}(k, \ell)>\frac{c k \log k \cdot \log \log k \cdot \log \log \log \log k}{(\log \log \log k)^{2}}
$$

where $p^{\prime}(k, \ell)$ is the first prime $q$ with $q \equiv \ell(\bmod k)$. In his proof of the finiteness of $P$-integers Pomerance [8] used the Jacobsthal function to show that

$$
P(k) \geq\left(e^{\gamma}+o(1)\right) \varphi(k) \log k
$$

where $\gamma$ is Euler's constant.

In our proofs we applied different tools. We use that the primitive residues modulo $k$ between 0 and $k$ are symmetric around $k / 2$. Our arguments are based on results about the zeros of the Riemann zeta function and estimates for the number of primes in intervals.

\section{LEMMAS}

Throughout the paper, let $p_{1}<p_{2}<\ldots$ be the increasing sequence of prime numbers. For any $x>1$, let $\pi(x)$ denote the number of prime numbers not exceeding $x$, and $\operatorname{Li}(x)=\lim _{\epsilon \rightarrow 0^{+}} \int_{t=0}^{1-\epsilon} \frac{d t}{\log t}+\int_{t=1+\epsilon}^{x} \frac{d t}{\log t}$. We put $\pi(x)=0$ for $0 \leq x \leq 1$.

Lemma 2.1. For any $x \in \mathbb{R}$ and $n \in \mathbb{N}$ we have

(i) $\pi(x)>\frac{x}{\log x}+\frac{x}{\log ^{2} x}+\frac{1.8 x}{\log ^{3} x}$ for $x>32299$;

(ii) $\pi(x)<\frac{x}{\log x}+\frac{x}{\log ^{2} x}+\frac{2.51 x}{\log ^{3} x}$ for $x>355991$;

(iii) $|\pi(x)-\operatorname{Li}(x)|<.4394 \frac{x}{(\log x)^{3 / 4}} \exp \left(-\sqrt{\frac{\log x}{9.646}}\right)$ for $x \geq 58$; 
(iv) if the Riemann Hypothesis holds, then $|\pi(x)-\operatorname{Li}(x)|<\frac{1}{8 \pi} \sqrt{x} \log x$ for $x>2656$;

(v) $\operatorname{Li}(x)>\pi(x)$ for $x \leq 10^{14}$;

(vi) $p_{n}<n(\log n+\log \log n)$ for $n \geq 6$;

(vii) $p_{n}>n \log n$ for $n \geq 1$;

(viii) $\frac{n}{\varphi(n)}<1.7811 \log \log n+\frac{2.51}{\log \log n}$ for $n \geq 3$.

Proof. We mention the references where the estimates from Prime Number Theory given in the lemma can be found.

(i), (ii) Dusart [2], p. 36.

(iii) Dusart [2], p. 41.

(iv) Schoenfeld [14], p. 339.

(v) Kotnik [6], p. 59.

(vi), (vii) Rosser and Schoenfeld [10], p. 69.

(viii) Rosser and Schoenfeld [10], p. 72.

Lemma 2.2. Let $x$ be a real number with $x>712000$. Then we have

$$
2 \pi\left(\frac{x}{2}\right)-\pi(x)>\frac{.693 x}{\log ^{2} x} .
$$

Proof. We have, by Lemma 2.1 (i), (ii), for $x>712000$,

$$
\begin{gathered}
2 \pi(x / 2)-\pi(x)> \\
\frac{x}{\log (x / 2)}+\frac{x}{\log ^{2}(x / 2)}+\frac{1.8 x}{\log ^{3}(x / 2)}-\frac{x}{\log x}-\frac{x}{\log ^{2} x}-\frac{2.51 x}{\log ^{3} x}> \\
\frac{x}{\log x\left(1-\frac{\log 2}{\log x}\right)}-\frac{x}{\log x}+\frac{x}{\log ^{2} x\left(1-\frac{\log 2}{\log x}\right)^{2}}-\frac{x}{\log ^{2} x}-\frac{.71 x}{\log ^{3} x}> \\
\frac{x}{\log x} \cdot \frac{\log 2}{\log x}+\frac{x}{\log ^{2} x} \cdot \frac{2 \log 2}{\log x}-\frac{.71 x}{\log ^{3} x}>\frac{.693 x}{\log ^{2} x} .
\end{gathered}
$$

Lemma 2.3. Let $x$ and $y$ be positive real numbers with $x>y, x \geq 59$. Then

$$
\begin{gathered}
2 \pi(x+y)-\pi(x)-\pi(x+2 y)> \\
\frac{y^{2}}{(x+2 y) \log ^{2}(x+2 y)}-\frac{1.7576(x+2 y)}{(\log x)^{3 / 4}} e^{-\sqrt{\frac{\log x}{9.646}}} .
\end{gathered}
$$

Proof. By Lemma 2.1 (iii),

$$
\begin{gathered}
2 \pi(x+y)-\pi(x)-\pi(x+2 y)> \\
2 \operatorname{Li}(x+y)-\operatorname{Li}(x)-\operatorname{Li}(x+2 y)-1.7576 \frac{x+2 y}{(\log x)^{3 / 4}} \exp \left(-\sqrt{\frac{\log x}{9.646}}\right) .
\end{gathered}
$$


Observe that

$$
\begin{gathered}
2 \operatorname{Li}(x+y)-\operatorname{Li}(x)-\operatorname{Li}(x+2 y)=\int_{x}^{x+y} \frac{d t}{\log t}-\int_{x+y}^{x+2 y} \frac{d t}{\log t} \\
=\int_{x}^{x+y}\left(\frac{1}{\log t}-\frac{1}{\log (t+y)}\right) d t=\frac{y^{2}}{\xi \log ^{2} \xi}
\end{gathered}
$$

for some $\xi$ with $x<\xi<x+2 y$, by the mean value theorem applied twice. Thus

$$
\begin{gathered}
2 \pi(x+y)-\pi(x)-\pi(x+2 y)> \\
\frac{y^{2}}{(x+2 y) \log ^{2}(x+2 y)}-1.7576 \frac{x+2 y}{(\log x)^{3 / 4}} \exp \left(-\sqrt{\frac{\log x}{9.646}}\right) .
\end{gathered}
$$

Lemma 2.4. Suppose the Riemann Hypothesis holds true.

Let $x>y>0, x \geq 2657$. Then

$$
\begin{gathered}
2 \pi(x+y)-\pi(x)-\pi(x+2 y)> \\
\frac{y^{2}}{(x+2 y) \log ^{2}(x+2 y)}-\frac{\log (x+2 y)}{\theta} \sqrt{x+2 y}
\end{gathered}
$$

where

$$
\theta=\left\{\begin{array}{l}
2 \pi \text { if } x+2 y>10^{14} \\
4 \pi \text { if } x+2 y \leq 10^{14} .
\end{array}\right.
$$

Proof. By Lemma 2.1 (iv), (v),

$$
\begin{gathered}
2 \pi(x+y)-\pi(x)-\pi(x+2 y)> \\
2 \operatorname{Li}(x+y)-\operatorname{Li}(x)-\operatorname{Li}(x+2 y)-\frac{\log (x+2 y)}{\theta} \sqrt{x+2 y} .
\end{gathered}
$$

The lemma follows in the same way as in the proof of Lemma 2.3.

\section{A CRiterion For an integer $k$ to Be not a P-Integer}

Suppose $k$ is a $P$-integer $>30$. Further, due to results from [3] and [12] mentioned in the introduction, we may also assume that neither $k$ nor $k / 2$ is prime. Let $\varphi(k)+\omega(k)=T$. Then there are exactly $\varphi(k)$ primes belonging to the set $\left\{p_{1}, \cdots, p_{T}\right\}$ which are coprime to $k$ and form a reduced residue system $\bmod k$. The remaining $\omega(k)$ primes in this set divide $k$. Let

$$
\begin{aligned}
D_{k}^{\prime} & =\left\{i \leq T: p_{i}(\bmod k)<\frac{k}{2}\right\}, \\
D_{k}^{\prime \prime} & =\left\{i \leq T: p_{i}(\bmod k) \geq \frac{k}{2}\right\}
\end{aligned}
$$


and

$$
D_{k}^{\prime \prime \prime}=\left\{i \leq T: p_{i} \mid k\right\} .
$$

Note that $\left|D_{k}^{\prime \prime \prime}\right|=\omega(k)$ where $|A|$ denotes the number of elements of a set $A$. By the symmetry of the primitive residues about $k / 2$, we get

$$
\left|D_{k}^{\prime} \backslash D_{k}^{\prime \prime \prime}\right|=\left|D_{k}^{\prime \prime} \backslash D_{k}^{\prime \prime \prime}\right|
$$

which implies

$$
\left|D_{k}^{\prime}\right|-\left|D_{k}^{\prime \prime}\right| \leq\left|D_{k}^{\prime \prime \prime}\right|=\omega(k) .
$$

Let $t$ be an integer such that $t k<p_{T}<(t+1) k$. We observe that if $p_{T} \in\left(t k, t k+\frac{k}{2}\right)$ we have

$$
\begin{gathered}
\left|D_{k}^{\prime}\right|=\sum_{n=0}^{t-1}\left(\pi\left(n k+\frac{k}{2}\right)-\pi(n k)\right)+T-\pi(t k), \\
\left|D_{k}^{\prime \prime}\right|=\sum_{n=0}^{t-1}\left(\pi(n k+k)-\pi\left(n k+\frac{k}{2}\right)\right)
\end{gathered}
$$

and if $p_{T} \in\left(t k+\frac{k}{2}, t k+k\right)$, then

$$
\begin{gathered}
\left|D_{k}^{\prime}\right|=\sum_{n=0}^{t}\left(\pi\left(n k+\frac{k}{2}\right)-\pi(n k)\right) \\
\left|D_{k}^{\prime \prime}\right|=\sum_{n=0}^{t-1}\left(\pi(n k+k)-\pi\left(n k+\frac{k}{2}\right)\right)+T-\pi\left(t k+\frac{k}{2}\right) .
\end{gathered}
$$

Thus we get

$$
\left|D_{k}^{\prime}\right|-\left|D_{k}^{\prime \prime}\right|=\sum_{n=0}^{t-1}\left(2 \pi\left(n k+\frac{k}{2}\right)-\pi(n k)-\pi(n k+k)\right)+T-\pi(t k)
$$

in the former case, and in the latter case

$$
\left|D_{k}^{\prime}\right|-\left|D_{k}^{\prime \prime}\right|=\sum_{n=0}^{t}\left(2 \pi\left(n k+\frac{k}{2}\right)-\pi(n k)-\pi(n k+k)\right)+\pi(t k+k)-T .
$$

Let $L(k)=t-1$ in the former case and $L(k)=t$ in the latter. Let $L:=L(k)$. We shall use this parameter $L$ later on without any further mentioning. Noting that $T-\pi(t k)$ and $\pi(t k+k)-T$ are both nonnegative and that $\omega(k)<\log k$, we find by (1) the following criterion.

Lemma 3.1. The integer $k$ is not a P-integer, if

$$
S_{L}:=\sum_{n=0}^{L}\left(2 \pi\left(n k+\frac{k}{2}\right)-\pi(n k)-\pi(n k+k)\right)-\log k>0 .
$$


We note that

$$
t k<p_{T} \leq p_{k} \leq k \log (k \log k)
$$

by Lemma 2.1 (vi). Thus

$$
L \leq t<\log (k \log k)
$$

On the other hand, using Lemma 2.1 (vii), (viii), putting $h(k)=$ $1.7811 \log \log k+\frac{2.51}{\log \log k}$, we get

$$
L+2 \geq t+1>\frac{p_{T}}{k} \geq \frac{p_{\varphi(k)}}{k}>\frac{\log k-\log h(k)}{h(k)} .
$$

\section{A computational Result}

Theorem 4.1. If $30<k \leq 10^{11}$, then $k$ is not a P-integer. Further, if $k$ is even with $30<k \leq 2 \cdot 10^{11}$ then $k$ is not a P-integer.

Proof. In [3] it has been computationally verified that no integer $k$ with $30<k<5.5 \cdot 10^{5}$ is a $P$-integer. Hence we may assume henceforth that

$$
5.5 \cdot 10^{5} \leq k \leq 2 \cdot 10^{11}
$$

To cover this interval, we apply a modified version of the algorithm used in [3].

To prove the statement for a given $k$ we apply the following strategy. We find a prime $p$ such that $k<p<p_{\varphi(k)}$ and $p(\bmod k)$ is also a prime. Then $k$ is not a $P$-integer. To make this strategy work on the whole range for $k$ under consideration, we shall make use of the following two properties. Let $k$ be an integer with $k \geq 5.5 \cdot 10^{5}$. Then we have

$$
\pi(k+1)+100<\varphi(k)
$$

and

$$
p_{\pi(k+1)+100}<1.5 k .
$$

These assertions can be easily checked e.g. by Magma [1], using parts (ii), (vi), (viii) of Lemma 2.1.

First we prove the statement for the even values of $k$. This is done by the algorithm below, which is based on the strategy indicated above.

Initialization. Let $k_{0}=5.5 \cdot 10^{5}$. Let $H$ be the list of the first 100 primes larger than $k_{0}+1$, i.e. $H=\left[p_{\pi\left(k_{0}+1\right)+1}, \ldots, p_{\pi\left(k_{0}+1\right)+100}\right]$.

Step 1. Check successively for the primes $p \in H$ whether $p\left(\bmod k_{0}\right)$ is also a prime. When such a $p$ is found then, by (4), $k_{0}$ is not a $P$-integer - proceed to the next step. 
Step 2. Check if $k_{0}+3$ is a prime. If not, then proceed to Step 3 . If so, this is the first element of $H$. Remove this prime from $H$, and append to $H$ the prime $p_{\pi\left(k_{0}+1\right)+101}$ which is the next prime to the last element of $H$.

Step 3. If $k_{0}<2 \cdot 10^{11}$ then put $k_{0}:=k_{0}+2$, and go to Step 1 .

Using this procedure we could check by a Magma program that there is no even $P$-integer in the interval $\left[5.5 \cdot 10^{5}, 2 \cdot 10^{11}\right]$.

Let now $k$ be odd with $5.5 \cdot 10^{5}<k<10^{11}$. Then by our algorithm above, using (4) and (5), we know that there exists a prime $p$ satisfying $2 k<p<\min \left\{3 k, p_{\varphi(2 k)}\right\}$ such that $q:=p(\bmod 2 k)$ is also a prime. Observe that $q<k$. Thus, as $\varphi(k)=\varphi(2 k), p$ is a prime such that $k<p<p_{\varphi(k)}$ and $q=p(\bmod k)$ is also a prime. Hence $k$ is not a $P$-integer and the theorem follows.

\section{Proofs of Theorems 1.1 And 1.2}

Proof of Theorem 1.1. Let $k$ be an integer with $k \geq 10^{3500}$. Then by (3), $L>500$. We apply Lemma 2.1 (i), (ii) to get

$$
\begin{gathered}
2 \pi(k / 2)-\pi(k)> \\
\frac{k}{\log (k / 2)}+\frac{k}{\log ^{2}(k / 2)}+\frac{1.8 k}{\log ^{3}(k / 2)}-\frac{k}{\log k}-\frac{k}{\log ^{2} k}-\frac{2.51 k}{\log ^{3} k} .
\end{gathered}
$$

For $n \geq 1$ we apply Lemma 2.3 with $x=n k, y=k / 2$ to find

$$
\begin{gathered}
2 \pi(n k+k / 2)-\pi(n k)-\pi(n k+k)> \\
\frac{k}{4(n+1) \log ^{2}(n k+k)}-1.7576 \frac{n k+k}{(\log n k)^{3 / 4}} \exp \left(-\sqrt{\frac{\log (n k)}{9.646}}\right)
\end{gathered}
$$

Put

$$
\begin{gathered}
f_{0}(k):=\frac{k}{\log \frac{k}{2}}+\frac{k}{\log ^{2} \frac{k}{2}}+\frac{1.8 k}{\log ^{3} \frac{k}{2}}-\frac{k}{\log k}-\frac{k}{\log ^{2} k}-\frac{2.51 k}{\log ^{3} k}-\log k, \\
f_{n}(k):=\frac{k}{4(n+1) \log ^{2}(n k+k)}-1.7576 \frac{n k+k}{(\log n k)^{3 / 4}} \exp \left(-\sqrt{\frac{\log (n k)}{9.646}}\right)
\end{gathered}
$$

for $n \geq 1$. A simple calculation shows that $S_{L}$, defined in Lemma 3.1, satisfies

$$
S_{L} \geq f_{0}(k)+\sum_{n=1}^{L} f_{n}(k)>0
$$

for $L \leq 1500$. This shows that $k$ is not a $P$-integer for such $L$. Hence we may assume that $L>1500$. 
We first check by Maple that $f_{n}(k)$ is a strictly monotone decreasing function of $n$. By (2) it is therefore enough to show that

$$
f_{0}(k)+\sum_{i=1}^{1500} f_{i}(k)+(L-1500) f_{n}(k)>0
$$

for $k=10^{3500}$ and $n=\lfloor\log (k \log k)\rfloor$. We check this again with Maple to get the final contradiction.

Remark. The constant 9.646 which occurs in Lemma 2.1 (iii) originates from a zero-free region of the Riemann-zeta function derived by Rosser and Schoenfeld ([11] Theorem 11), where the constant appears as $R$. The zero-free region has been widened by Kadiri [5] where the corresponding constant $R$ is 5.69693. If this constant would be substituted into Lemma 2.1 (iii) instead of the constant 9.646 and we follow our argument, we obtain that if $k$ is a $P$-integer, then $k<10^{1000}$. However, we do not know if this substitution is justified.

Proof of Theorem 1.2. Suppose the Riemann Hypothesis is true. Let $k$ be an integer with $k \geq 3 \cdot 10^{13}$. By Lemma 2.2, we get

$$
2 \pi\left(\frac{k}{2}\right)-\pi(k)>\frac{.693 k}{\log ^{2} k}>\log k>\omega(k) .
$$

For $n=1,2, \ldots,\lfloor\log (k \log k)\rfloor-1$ we apply Lemma 2.4 with $x=n k$, $y=k / 2$ to find

$$
\begin{gathered}
2 \pi\left(n k+\frac{k}{2}\right)-\pi(n k)-\pi(n k+k)> \\
\frac{k}{4(n+1) \log ^{2}(n k+k)}-\frac{\log (n k+k)}{2 \pi} \sqrt{n k+k} .
\end{gathered}
$$

The term on the right hand side of the above inequality is positive if

$$
\pi \sqrt{k}>2(n+1)^{1.5} \log ^{3}(n k+k) .
$$

This is satisfied, since $n<\log (k \log (k))-1$ and $k \geq 3 \cdot 10^{13}$. Hence by Lemma 3.1, we find that $k$ is not a $P$-integer.

Next we take $k<3 \cdot 10^{13}$. By Theorem 4.1, we may assume $k>10^{11}$. Note that $L<\log (k \log k) \leq 34$. Further

$$
L<\log k+\log \log k<1.13 \log k
$$

giving

$$
k>e^{.88 L}>10^{.38 L} \text {. }
$$

Define

$$
k_{L}=\left[10^{\{.38 L\}}\right] 10^{[.38 L]} .
$$


where $[x]$ and $\{x\}$ denote the integral and fractional part of any real number $x$. Note that for any fixed $L$ with $L \leq 34$ if $L(k) \geq L$, then $k \in\left[k_{L}, 3 \cdot 10^{13}\right)$. Applying Lemma 2.4 with $x=n k, y=k / 2$ we find

$$
\begin{gathered}
S_{L}>2 \pi(k / 2)-\pi(k)+ \\
+\sum_{n=1}^{L}\left(\frac{k}{4(n+1) \log ^{2}(n k+k)}-\frac{\log (n k+k)}{4 \pi} \sqrt{n k+k}\right) .
\end{gathered}
$$

For $n=1, \ldots, L$, put

$$
\begin{array}{r}
F_{n}(k):=\frac{1}{L}\left(\frac{k}{\log (k / 2)}+\frac{k}{\log ^{2}(k / 2)}+\frac{1.8 k}{\log ^{3}(k / 2)}\right) \\
-\frac{1}{L}\left(\frac{k}{\log k}+\frac{k}{\log ^{2} k}+\frac{2.51 k}{\log ^{3} k}+\log k\right) \\
+\frac{k}{4(n+1) \log ^{2}(n k+k)}-\frac{\log (n k+k)}{4 \pi} \sqrt{n k+k} .
\end{array}
$$

We have, by Lemma 2.1 (i), (ii),

$$
S_{L}-\log k>\sum_{n=1}^{L} F_{n}(k) .
$$

So it is sufficient to show that the right hand side is positive. For this, we proceed as follows. First, let $29 \leq L \leq 34$. We calculate the value $k_{L}$ from its definition above. Thus $\left(L, k_{L}\right)$ is one of the pairs from

$\left\{\left(29,10^{11}\right),\left(30,2 \cdot 10^{11}\right),\left(31,6 \cdot 10^{11}\right),\left(32,10^{12}\right),\left(33,3 \cdot 10^{12}\right),\left(34,8 \cdot 10^{12}\right)\right\}$.

We check by Maple that all functions $F_{n}(k)$ are strictly monotone increasing on $\left[k_{L}, 3 \cdot 10^{13}\right]$, and further

$$
\sum_{n=1}^{L} F_{n}\left(k_{L}\right)>0 .
$$

Hence by Lemma 3.1, there is no P-integer $k$ with $L(k) \in[29,34]$. Now we consider $k \in\left[10^{11}, 3 \cdot 10^{13}\right]$. Then obviously $L(k)>0$. We may assume $1 \leq L \leq 28$. We check that all functions $F_{n}(k)$ are strictly monotone increasing and the preceding inequality also holds. Hence we conclude that no integer $k \in\left[10^{11}, 3 \cdot 10^{13}\right]$ is a $P$-integer.

\section{AcKnowledgements}

The authors are grateful to the referee for her/his helpful and useful remarks. 


\section{REFERENCES}

[1] W. Bosma, J. Cannon, C. Playoust, The Magma algebra system. I. The user language, J. Symbolic Comput. 24 (1997), 235-265.

[2] P. Dusart, Autour de la fonction qui compte le nombre de nombres premiers, Thèse, Université de Limoges, 1998, 172 pp.

[3] L. Hajdu and N. Saradha, On a problem of Recaman and its generalization, J. Number Theory 131 (2011), 18-24.

[4] D. R. Heath-Brown, Zero-free regions for Dirichlet L-functions, and the least prime in an arithmetic progression, Proc. London Math. Soc. 64(3) (1992), 265-338.

[5] H. Kadiri, Une région explicite sans zéros pour la fonction $\zeta$ de Riemann, Acta Arith. 117 (2005), 303-339.

[6] T. Kotnik, The prime-counting function and its analytic approximations, Adv. Comput. Math. 29 (2008), 55-70.

[7] Yu. V. Linnik, On the least prime in an arithmetic progression, Rec. Math. (Mat. Sbornik) N.S. 15 (57) (1944), 139-178 (347-368).

[8] C. Pomerance, A note on the least prime in an arithmetic progression, J. Number Theory 12 (1980), 218-223.

[9] K. Prachar, Über die kleinste Primzahl einer arithmetischen Reihe, J. Reine Angew. Math. 206 (1961), 3-4.

[10] J.B. Rosser, L. Schoenfeld, Approximate formulas for some functions of prime numbers, Illinois J. Math. 6 (1962), 64-94.

[11] J.B. Rosser, L. Schoenfeld, Sharper bounds for the Chebyshev functions $\theta(x)$ and $\psi(x)$, Math. Comput. 29 (1975), Number 129, 243-269.

[12] N. Saradha, Conjecture of Pomerance for some even integers and odd primorials, Publ. Math. Debrecen (to appear).

[13] A. Schinzel, Remark on the paper of K. Prachar "Über die kleinste Primzahl einer arithmetischen Reihe", J. Reine Angew. Math. 210 (1962), 121-122.

[14] L. Schoenfeld, Bounds for the Chebyshev Functions $\theta(x)$ and $\psi(x)$. II, Math. Comput. 30 (1976), Number 134, 337-360.

[15] Triantafyllos Xylouris, On Linnik's constant, Thesis, arXiv:0906.2749 [math.NT], 2009. 
L. HAJDU

University of DeBrecen,

InSTITUTE OF MATHEMATICS,

and the Number Theory Research Group of the Hungarian Academy

OF SCIENCES,

P.O. Box 12.,

H-4010 DEBRECEN,

HUNGARY

E-mail address: hajdul@science.unideb.hu

N. SARADHA

SChool of Mathematics,

Tata Institute of Fundamental Research,

Dr. HomibhabHa Road,

Colaba, Mumbai,

INDIA

E-mail address: saradha@math.tifr.res.in

R. TIJDEMAN

Mathematical Institute

LEIDEN UNIVERSITY

P.O.Box 9512

2300 RA LEIDEN

The Netherlands

E-mail address: tijdeman@math.leidenuniv.nl 